\title{
Compact Steep-Spectrum Radio Sources and Ambient Medium Density
}

\section{Ezeugo Jeremiah Chukwuemerie}

Department of Physics and Industrial physics, NnamdiAzikiwe University, Awka, Anambra State, Nigeria

Email address:

chuksemeria@yahoo.com

To cite this article:

Ezeugo Jeremiah Chukwuemerie. Compact Steep-Spectrum Radio Sources and Ambient Medium Density. International Journal of Astrophysics and Space Science. Vol. 3, No. 1, 2015, pp. 1-6. doi: 10.11648/j.ijass.20150301.11

\begin{abstract}
Analytical and statistical methods, with some plausible assumptions, have been used in this work to show presence of dense medium around compact steep-spectrum sources in our sample. This is done by carrying out regression analyses using our estimated jet's internal densities $\left(n_{j}\right)$ and some other source parameters for possible deductions. Results indicate that $n_{j}-D$ and $n_{j}-n_{e}$ data (where $D$ and $n_{e}$ are respectively, observed source linear size and ambient medium density) show strong correlations and yield power-law relations of the forms, $n_{j} \sim D^{-3.54 \pm 0.02}$ and $n_{j} \sim n_{e}^{1.68 \pm 0.08}$. A comparison with obtained theoretical relations suggestively indicates presence of dense gases around these sources. In addition, a simple linear regression analysis of $v_{p}-n_{j}$ data shows that jet's particles may be responsible (in addition to the ambient gases) for the observed spectral turnover characteristic of compact steep spectrum sources.
\end{abstract}

Keywords: Spectral Turnover, Steep-Spectrum, Astrophysical Jet, Ambient Density, Active Galaxies

\section{Introduction}

Compact steep spectrum sources (CSSs) contain special characteristics that make them be considered as a separate class of objects in addition to the conventional radio sources which consist mainly of radio galaxies and radio loud quasars $[1,2,3,4,5]$. Similarly, CSS sources are made up of radio galaxies and radio loud quasars but on sub-galactic dimensions. Their projected linear sizes, $D$, range as follows: $D \leq 20 \mathrm{kpc}$ assuming Hubble constant, $H_{0}=75 \mathrm{~km}^{-\mathrm{s}} \mathrm{Mpc}^{-1}$, and deceleration parameter, $q_{0}=0.0$. Their radio luminosity, $P$, at $5 \mathrm{GHz}$ is greater than $10^{25.5} \mathrm{WHz}^{-1}[2,3,6,7]$. They show double, triple and core-jet morphologies on radio maps [3]. They have steep high frequency spectrum of spectral index, $\alpha \geq 0.5\left(S_{v} \sim v^{-\alpha}\right)$; where $S_{v}$ is flux density and $v$ is observing frequency). Their spectral turnover is believed to be due to synchrotron self-absorption in a compact component with a large magnetic field. Generally, they have very low polarization at both radio and optical bands, usually, less than one percent $[2,3,8,9]$. Their proportion is high; from about $15 \%$ to $30 \%$, depending on the selection frequency, among distant $(z>0.2)$ radio sources of high luminosity $[2,3]$.

Usually, the structure of a typical extragalactic radio source takes the form of a central core (which, more or less, coincides with the nucleus of the host galaxy) straddled by two-sided jets. At the end of each jet is located a radio-emitting lobe. The jet is believed to serve as a conduit through which jet's materials feed the lobe. However, it is believed that from accretion disks much of the energy powering the jets is derived $[4,10]$. Gravitational field of a suppermassive black hole, believed to reside at the central core of a radio source, attracts gas clouds in its vicinity[11]. This causes the gas clouds to accelerate toward the black hole from a range of directions and collide with each other as they approach their orbital trajectory, and finally form an accretion disk. This accretion disk is believed to be the source of jet's materials $[4,10,11]$, but the process by which this occurs is not yet fully understood. However, one of the factors that appear to affect the propagation of a jet is jet's internal density (or ratio of jet's internal density to ambient medium density) [12].

Therefore, the purpose of this work is to derive a mathematical relation for estimating internal densities of magnetically driven jets; and use the estimates, through simple regression analyses, to find how they correlate with some other source parameters for possible deductions. The regression analyses are based on the list of 61 CSS sources (with observed linear sizes, $D \leq 20 \mathrm{kpc}$ ) compiled by [3] from the 3CR and source samples from [8]. 


\section{Magnetic Force on Particles of Constant Velocity}

As pointed out by [10], one of the most promising models for jet's formation in AGN is the magnetic acceleration model $[13,14]$. Moreover, according to the study of magnetically driven jets [10], the terminal velocity of a jet is comparable to the rotational velocity of the disk at the foot of the jet. The implication is that a relativistic jet is produced at a position very close to the event horizon of a black hole.

Assuming particles moving with aconstant velocity, $V_{j}$, in a constant magnetic field, $B_{j}$, then the magnetic force, $F_{B}$, acting on such an ensemble ofparticles to produce a jetat the core of a typical radio source may be given by

$$
F_{B} \approx n e V_{j} B_{j}
$$

where $n$ is number of particles. Approximating it to a tangential force (whose result is the usual straightness of jets), (1) may be written as

$$
M_{j} a_{j} \approx n e V_{j} B_{j}
$$

where $M_{j}$ is mass of jet, $a_{j}$ is acceleration of jet, and $V_{j}$ now represents jet's terminal velocity.

By definition,power, $P_{c j}$, over the source dynamical age, $T$, may be given by

$$
P_{c j}=\frac{M_{j} a D}{T}
$$

where $D$ is taken to be the source linear size. Combining (2) and (3), $B_{j}$ becomes

$$
B_{j} \approx \frac{P_{c j} T}{n e V_{j} D}
$$

\section{Jet's Internal Density}

However, it is believed that the observed radio emission from jets and lobes, as well as, their confinement emanate from their interactions with the ambient media through ram pressure $[2,15,16,17]$. Therefore, we assume lobe confinement by ram-pressure balance with the external medium, and have $[2,15,16]$ :

$$
P_{i} \approx n_{e} m_{H} V_{L}^{2}
$$

where $P_{i}$ is lobe's internal pressure, $n_{e}$ is external (or ambient) medium density, $m_{H}$ is proton mass and $V_{L}$ is lobe velocity. Using the last equation, it can be shown that power, $P_{c j}$, delivered to the jet can be expressed according to the following relation $[16,18]$ :

$$
P_{c j}=m_{H} c n_{e} \Omega D^{2} V_{j}^{2}
$$

where $\Omega$ is jet's opening solid angle. Putting it in (4), we obtain

$$
B_{j} \approx \frac{m_{H} c n_{e} \Omega \mathrm{DV}_{\mathrm{j}} \mathrm{T}}{n e}
$$

Furthermore, assuming a jet of constant radius, $R_{j}$, the number, $n$, of particles in a jet can be expressed in relation to the number density, $n_{j}$, of the jet's particles as

$$
n \approx n_{j} \pi R_{j}^{2} D
$$

However, [19] has pointed out that the correlations between spectral turnover $\left(v_{p}\right)$, flux density $\left(S_{v}\right)$, and source angular size $(\theta)$ imply a linear correlation between the lobes and source sizes; thereby, indicating that during the evolution of young radio sources, the ratio of the size of the lobes to the distance between the two lobes is constant. This implies that lobe's size increases as the linear size, $D$, of an extragalactic radio source increases. Similarly, assuming that source linear size and jet's width maintain a constant ratio, then jet's radius may appreciate with distance from the central core according to the relation,

$$
R_{j} \approx 0.004 D
$$

This is estimated by taking the ratio of the distance between the ends of the two hotspots and jet's width of 3C 405 (Cygnus A). Generalizing this for radio sources and combining (8) and (9) yields

$$
n \approx\left(1.6 \times 10^{-5}\right) n_{j} \pi D^{3}
$$

Putting it in (7), $B_{j}$ becomes

$$
B_{j} \approx \frac{m_{H} c n_{e} \Omega V_{j} T}{\left(1.6 \times 10^{-5}\right) \pi n_{j} e D^{2}}
$$

Hence, jet's internal density becomes

$$
n_{j} \approx \frac{m_{H} c n_{e} \Omega V_{j} T}{\left(1.6 \times 10^{-5}\right) \pi B_{j} e D^{2}}
$$

The last equation shows that

$$
n_{j} \sim D^{-2}
$$

implying that $n_{j}$ falls off as $D^{-2}$ from the central core of a radio source for constantjet's velocity and magnetic field.

Applying synchrotron-self absorption theory [12,19], source magnetic field, $B_{s}$, can be written as follows [12]:

$$
B_{S}=\frac{\pi^{3} \theta^{4} m_{e}^{3} v^{5}}{18 S_{\nu}^{2} e}
$$

$m_{e}$ is mass of an electron, $v$ is observing frequency, $\theta$ is source's angular size, and $v$ is flux density. For the purpose of estimating jet's internal density, we assume $B_{j} \approx B_{s}$. Therefore, combining (12) and (14), we obtain

$$
n_{j} \approx 1.125 \times 10^{6}\left(\frac{m_{H} c \Omega V_{j}}{\pi^{4} m_{e}^{3}}\right)\left(\frac{n_{e} T S_{v}^{2}}{D^{2} \theta^{4} v^{5}}\right)
$$

\section{Analyses and Result}

Taking typical values of the following quantities: $m_{H}=$ $1.67 \times 10^{-27} \mathrm{~kg}, \quad c=3 \times 10^{8} \mathrm{~ms}^{-1}, \Omega=3.6 \times 10^{-5} \mathrm{sr}$, $V_{j} \approx 0.3 c[10]$, and $m_{e}=9.109 \times 10^{-31} \mathrm{~kg}$, (15) becomes 


$$
n_{j} \approx 2.476 \times 10^{79}\left(\frac{n_{e} T S_{v}^{2}}{D^{2} \theta^{4} v^{5}}\right)
$$

We notice that the parameters at the right-hand side of the last equation are either observed or estimable. The source ages $(T)$ used in our analyses are estimated by dividing the individual source's observed linear size by lobe's velocity (0.251c) of the CSS source, 0710+439, observed by [19]. Since the estimated ages which range from $10^{2} \mathrm{Yr}$ to $10^{5} \mathrm{Yr}$ are consistent with those in the literature $[9,15,21]$, we assume this lobe's velocity for all the CSS sources in our sample.

Therefore, using the observed parameters (i.e. $\theta$ [where 1 arc second $\left.=4.85 \times 10^{-6} \mathrm{rad}\right], \quad v, D$ [where $1 \mathrm{pc}=$ $\left.3.086 \times 10^{16} \mathrm{~m}\right]$, and $S_{v}$ [where $\left.1 \mathrm{Jy}=10^{-26} \mathrm{Wm}^{-2} \mathrm{~Hz}^{-1}\right]$ ) from [3], the estimated source ages and ambient density $n_{e}$ obtained from [18], we estimate jet's density for each source.

Using these estimates, we plot $n_{j}-D$ data as shown in Fig. 1.
A simple linear regression analysis of the plot gives

$$
\log n_{j}=(-24.6 \pm 0.2)-(3.54 \pm 0.02) \log D
$$

with correlation coefficient, $r \approx 0.7$.

Also, we carry out a simple linear regression analysis of $n_{j}-n_{e}$ plot as shown in Fig. 2 ; where values of $n_{e}$ for the sources are obtained from [18]. Result gives

$$
\log n_{j}=(-23.0 \pm 0.2)+(1.68 \pm 0.08) \log n_{e}
$$

with $r \approx 0.8$.

Finally, we carry out a linear regression analysis of spectral turnover $\left(v_{p}\right)$ and jet's density (Fig. 4). This yields

$$
\log v_{p}=(4.8 \pm 0.2)+(0.09 \pm 0.01) \log n_{j}(19)
$$

with correlation coefficient, $r \approx 0.6$.

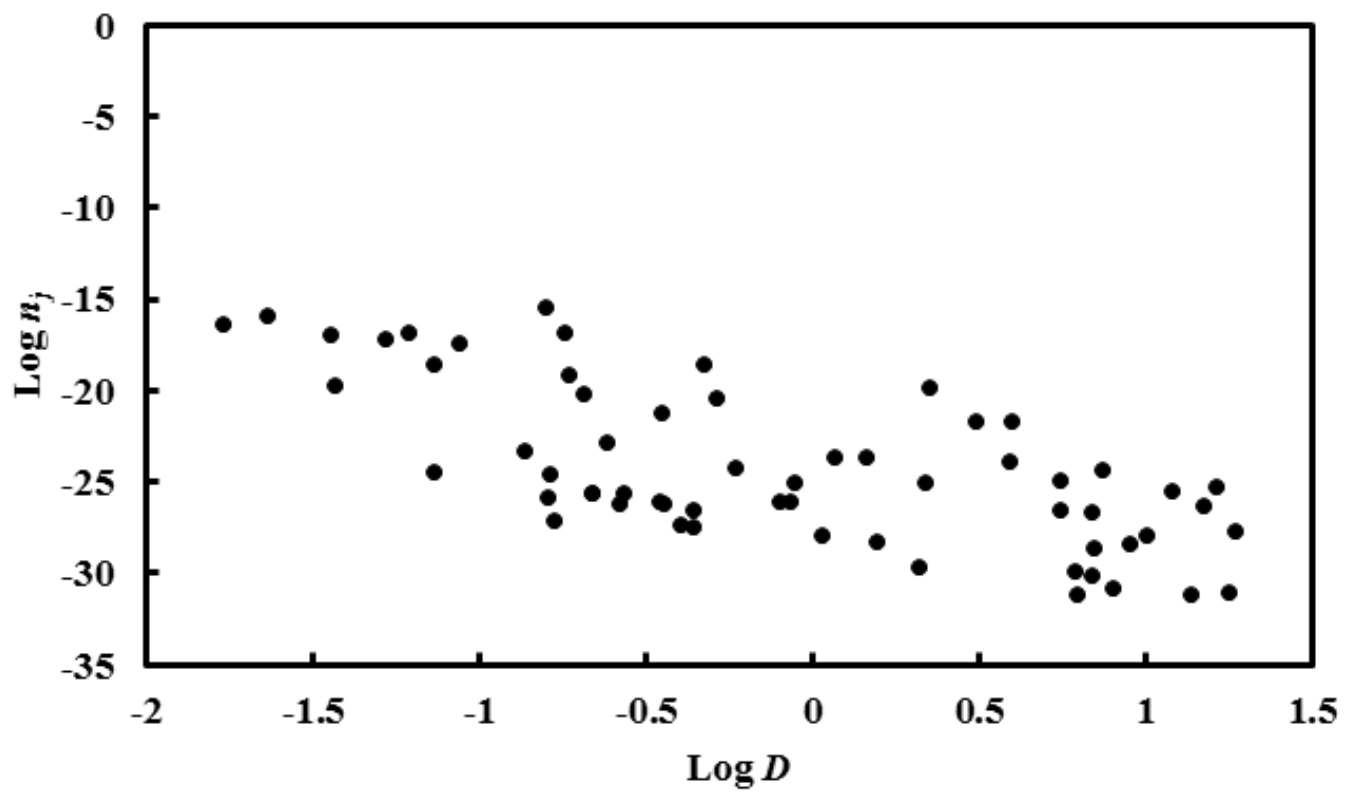

Figure 1. The scatter plot of jet's density against linear size.

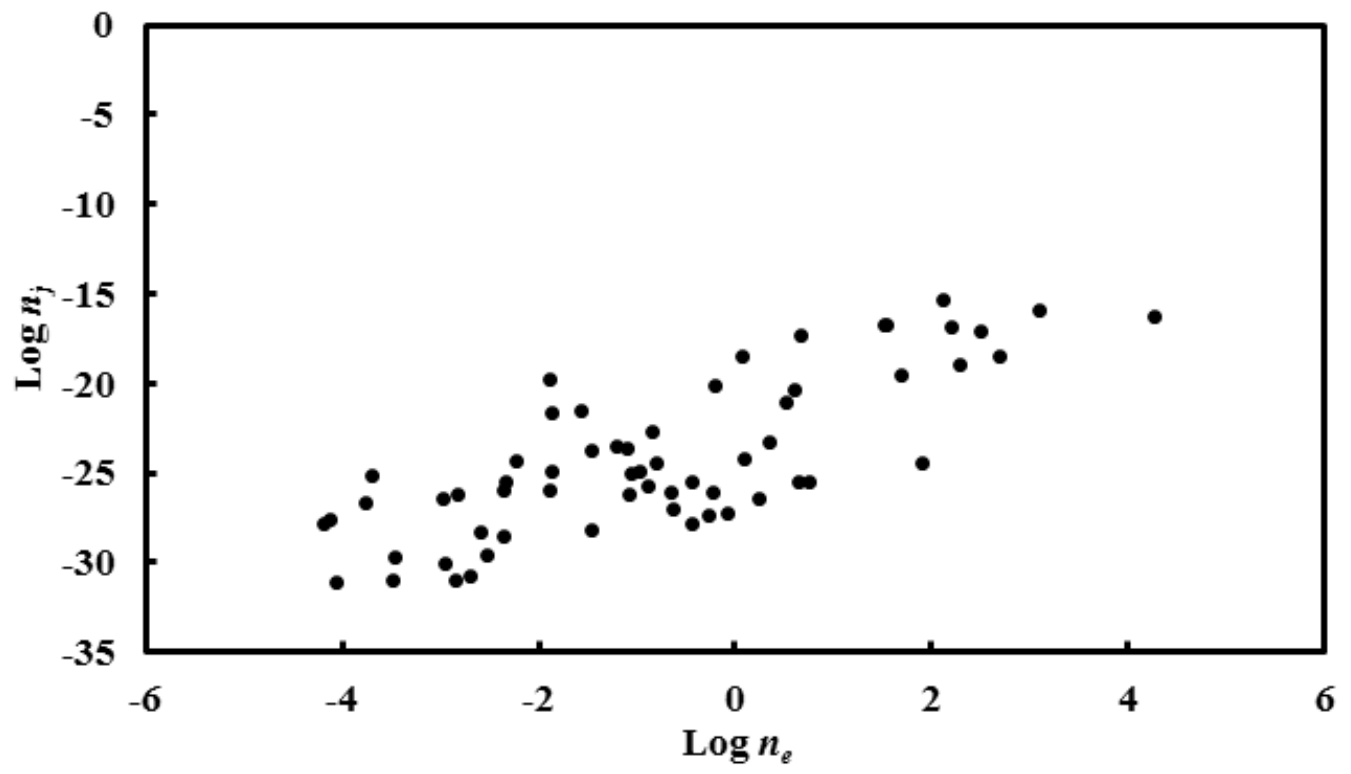

Figure 2. The scatter plot of jet's density against ambient density. 


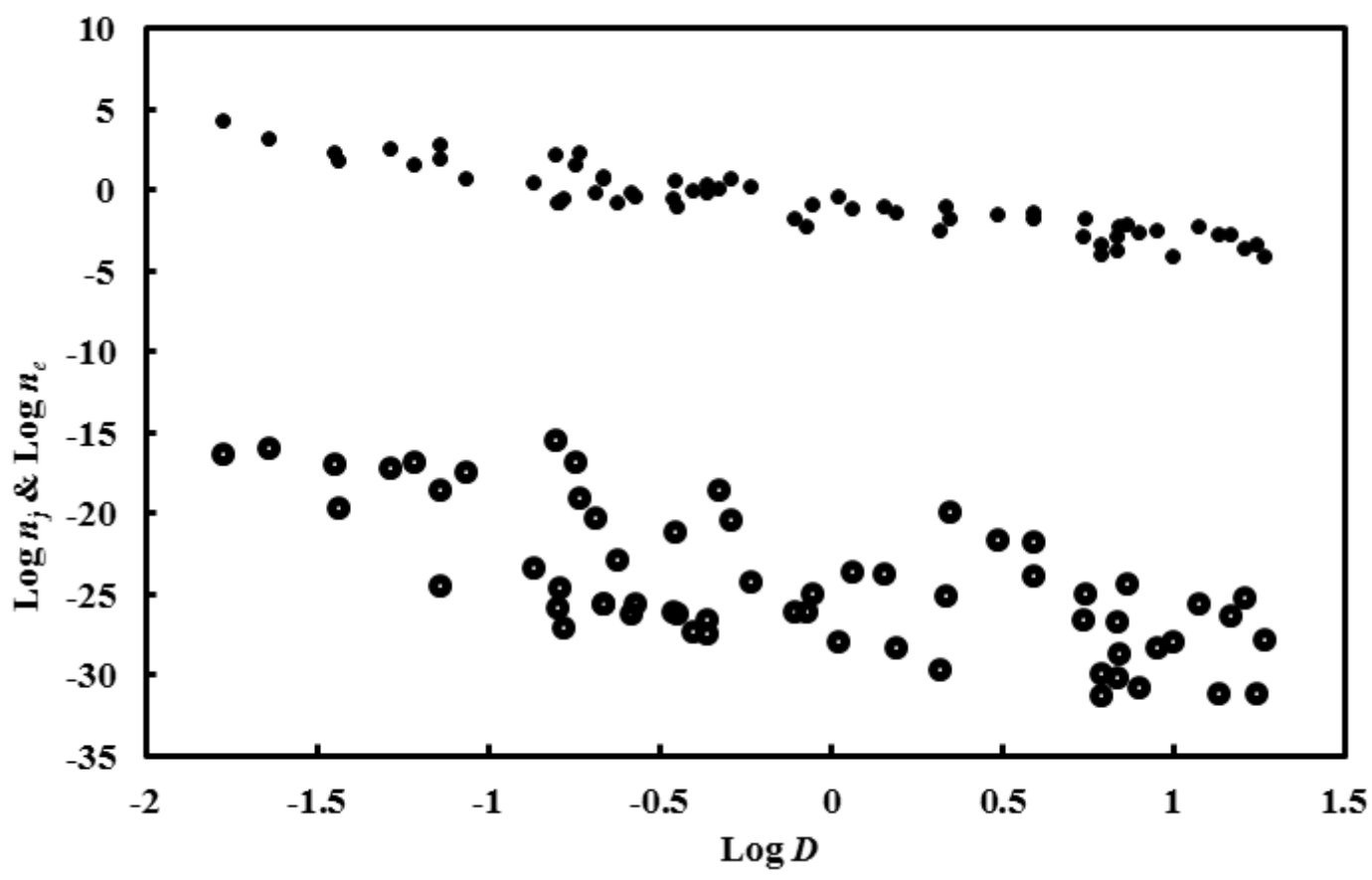

Figure 3. The scatter plot of $n_{j}$ (open circles) \& Log $n_{e}($ filled circles) against $D$.

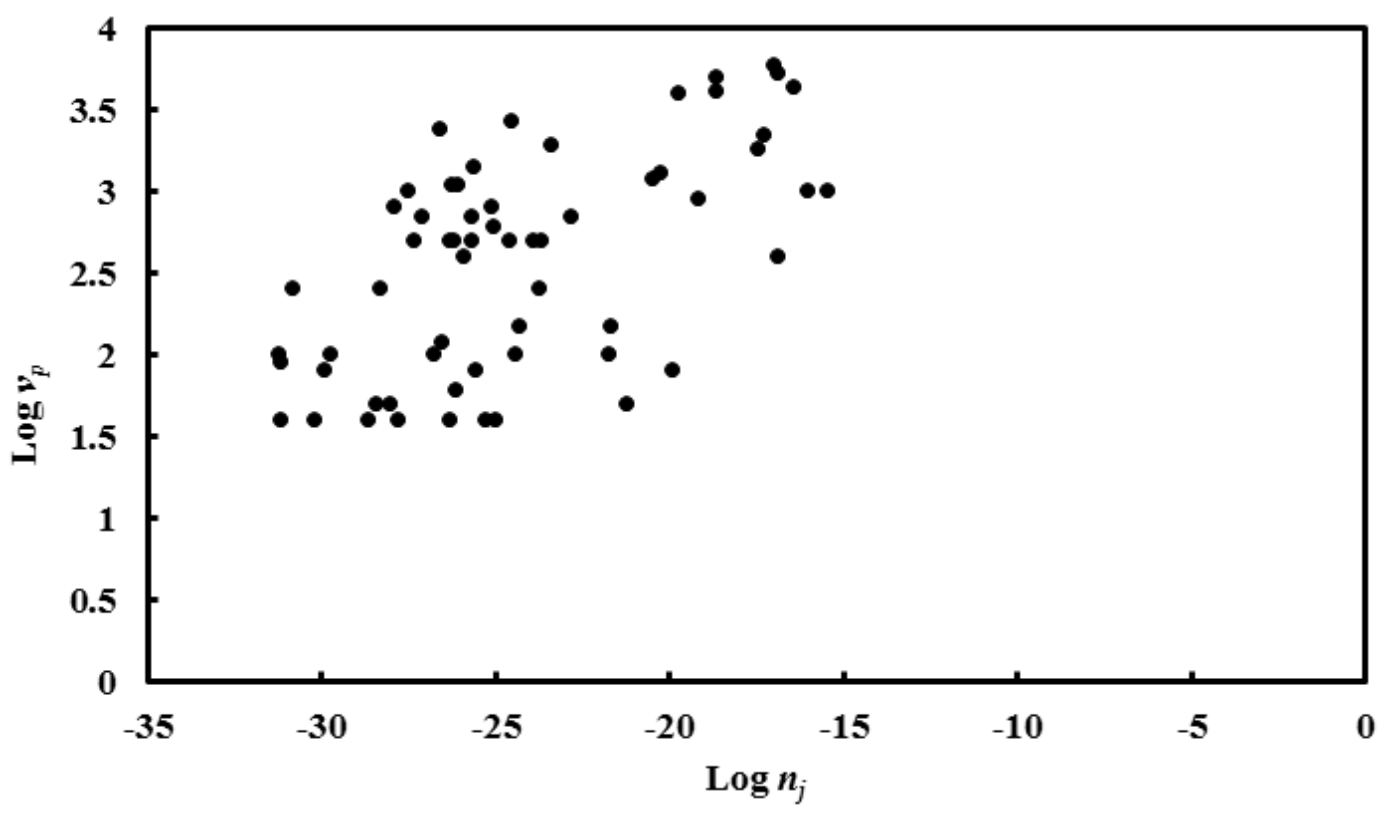

Figure 4. The scatter plot of spectral turnover against jet's density.

\section{Discussion}

In the previous sections, we find from theory (12) that for a source with constant magnetic force and constant particle's velocity $\left(V_{j}\right)$, jet's density $\left(n_{j}\right)$ is expected to decline with distance $(D)$ from the central core of an extragalactic radio source according to the relation, $n_{j} \sim D^{-\zeta_{1}}\left(\zeta_{1}=2.0\right)$. This implies that as distance from the core increases, some particles of jet fizzle out. If an ambient medium of low (or negligible) density is considered, the energy lost by jet's particles would be mainly outside interactions with particles of ambient medium; and hence, must be due to interactions with fields surrounding the source. However, in absence of any other radiation whose mechanism of production may not be in consonance with the foregoing, synchrotron radiation which can only result from particles' interactions with magnetic field appears to fit in. This is supported observationally, indicating that the radio continuum emission from jet shows spectra characteristic of synchrotron radiation $[3,9,19]$. Furthermore, if some jet's particles fizzle out as a result of energy losses due to synchrotron radiation, then jet's luminosity is also expected to drop with distance (see also $[3,22]$ ).

On the other hand, [18] has pointed out that the relation, $v_{p} \sim n_{e}^{0.35}$, between spectral turnover $\left(v_{p}\right)$ and ambient medium density $\left(n_{e}\right)$ suggests that the spectral turnover 
constitutes a characteristic signature of dense gases around CSS sources. Moreover, considering their estimated age range, $10^{2} \mathrm{Yr}-10^{5} \mathrm{Yr}$ (Table 1) (see also $[15,21]$ ), the relation implies that CSS sources might be young radio sources growing in dense ambient media. Therefore, if we assume a dense medium around a CSS source, then more jet's particles would fizzle out due to their appreciable interactions with particles of ambient medium (see [16,18,23,24,25]; and hence, the index $\left(\zeta_{1}\right)$ is expected to increase. This implies that a simple regression analysis of $n_{j}-D$ data should yield a slope (say, $\left.\zeta_{2}\right) \geq 2.0$.

Just as expected, $n_{j}-D$ data (Fig. 1) show a strong correlation (with $r \approx 0.7$ ), and yield $n_{j} \sim D^{-\zeta_{2}}$ (see equation (17)); where $\zeta_{2}(=3.54 \pm 0.02)$ is greater than $\zeta_{1}(=2.0)$. This implies that greater number of jet's particles than predicted in the theory fizzle out; and hence, suggests the presence of dense ambient media around CSS sources.

Moreover, (15) shows a theoretical relationship $\left(n_{j} \sim n_{e}^{\psi_{1}}\right.$, where $\left.\psi_{1}=1.0\right)$ between jet's density $\left(n_{j}\right)$ and ambient medium density $\left(n_{e}\right)$; and predicts that both densities decline from the core. However, the result of the regression analysis (18) of $n_{j}-n_{e}$ data (Fig. 2) shows, with strong correlation ( $r \approx 0.8$ ), that $n_{j} \sim n_{e}^{\psi_{2}}$; where $\psi_{2} \approx 1.68 \pm 0.08$. Though, it indicates a direct relationship between the two densities which is consistent with (15), the disparity in the two indices $\psi_{1}<\psi_{2}$ shows that $n_{j}$ declines faster than $n_{e}$; and hence, supports the suggestion that dense gases exist around CSS sources.

Fig. 3 shows $n_{e}-D$ (filled circles) and $n_{j}-D$ (open circles) plots on the same axes, indicating variations of the two densities over distances. The plot shows that as ambient medium density $\left(n_{e}\right)$ declines according to the relation, $n_{e} \sim D^{-2.2}[18,23,25]$, jet's density declines according to the relation, $n_{j} \sim D^{-3.5}$. Hence, $n_{j}$ declines faster than $n_{e}$. This may result from the interactions of jet's particles with ambient gases; therefore, indicating presence of dense ambient media around CSS sources.

Finally, Fig. 4 showsa plot between spectral turnover $\left(v_{p}\right)$ and jet's density $\left(n_{j}\right)$ with a power-law relation of the form,

$$
v_{p} \sim n_{j}^{\delta}
$$

where $\delta=0.09 \pm 0.01$ and $r \approx 0.6$. This is indicative that jet's particles (in addition to ambient gases as pointed out by [18]) may be responsible for the observed spectral turnover characteristic of compact steep spectrum sources.

\section{Conclusion}

We have shown a theoretical relation that may connect jet's internal density $\left(n_{j}\right)$ and observed linear size $(D)$, as well as, between $n_{j}$ and ambient medium density $\left(n_{e}\right)$. We have also shown that strong correlation exists between $n_{j}$ and $D$ (Fig. 1), as well as, between $n_{j}$ and $n_{e}$ (Fig. 2). The departures of the results of regression analyses of $n_{j}-D$ and $n_{j}-n_{e}$ data from their respectively obtained theoretical values, suggest the presence of dense gases around Compact Steep Spectrum sources. Moreover, we have seen from a simple linear regression analyses of spectral turnover $\left(v_{p}\right)$ and $n_{j}$ that the good correlation (with $r \approx 0.6$ ) suggestively indicates that jet's particles (in addition to the ambient gases; see [18]) causes spectral turnover which characterizes CSS sources.

\section{References}

[1] C. E. Akujor, andS. T.Garrington,"Compactsteep-spectrum sources - polarization observations at 1.6, 8.4 and $15 \mathrm{GHz}$,"Astron and Astrophy. Suppl. Ser.,vol. 122, pp. 235-255, 1995

[2] C. Fanti, R.Fanti, D. C.Dallacasa,R. T.Schilizzi,R. E.Spencer,and C.Stanghellini,"Are compactsteep-spectrum sources young?"Astron. and Astrophy., vol. 302, pp. 317-326, 1995

[3] C. P. O'Dea, "The compact steep-spectrum sources andGigahertz peaked-spectrum radio sources", Pub. Astron.Soc. Ppacific,vol. 110, pp. 493-532, 1998

[4] C. A. Jackson,"Radio source evolution and unifiedschemes," Publ. Astron. Soc. Aust., vol. 16, pp.124-129, 1999

[5] J. Machalski,M.Jamrozy,and D. J.Saikia,“A multifrequency study of giant radio sources III. Dynamicalage vs. spectral age of the lobes of selected sources",Mont. Not. Roy. Ast. Soc., vol. 395, pp. 812-824, 2009

[6] D. J. Saikia,S.Jeyakumar,P. J.Wiita,H. S.Sanghera,andR. E.Spencer, "Compact steep-spectrum radiosources and unification schemes," Mon. Not. Roy. Ast.Soc., vol. 276, pp. 1215-1223, 1995

[7] C. J. Stanghellini,D.Dallacasa,C. P. O'Dea,S. A.Baum,R. Fanti,C. Fanti,"VLBA observations ofGHz-peaked-spectrum radio sources at $15 \mathrm{GHz}$,"Astron. and Astrophy., vol. 377, pp. 377-388, 2001

[8] J. A. Peacock, andJ. V. Wall, "Bright extragalacticradio sources at 2.7GHz-II," Mont. Not. Roy. Ast. Soc.,vol. 198, pp. $843-860,1982$

[9] M. Murgia,R.Fanti, L.Gregorini, U. Klein,K. H.Mark,andM. Vigotti, "Synchrotron spectra and ages ofcompact steep spectrum radio sources,"Astron. andAstrophy., vol. 345, pp. 769-777, 1999

[10] S. Koide,K. Shibata,and T.Kudoh,"Relativistic jetformation from black hole magnetized accretion disks,",The Astrophy. Journ., vol. 522, pp.727-752, 1999

[11] A. Bongiorno, A. Merloni, M. Brusa, B. Magnelli, M. Salvato, M. Mignoli, G. Zamorani, F. Fiore, D. Rosario, V. Mainieri, H. Hao, A. Comastri, C. Vignali, I. Balestra, S. Bardelli, S. Berta, F. Civano, P. Kampczyk, E. Le Floc'h, E. Lusso, D. Lutz, L. Pozzetti, F. Pozzi, L. Riguccini, F. Shankar, andJ. Silverman, "Accreting supermassive black holes in the COSMOS field and the connection to their host galaxies,"Mon. Not. Roy Ast. Soc., vol. 427, pp. 3103-3133, 2012

[12] I. Robson, Active Galactic Nuclei, England: John Wileyand Sons, 1996, pp. 269-307

[13] R. D. Blandford,and D. G. Payne,"Hydromagneticflows from accretion discs and the production of radiojets,"Mont. Not. Roy. Ast. Soc., vol. 199, pp. 883-903,1982 
[14] G. Ustyugova,A. V. Voldoba,M. M.Romanova,V.M.Chechetkin, and R. V. E Lovelace,"Magnetohydrodynamic simulations of outflows from accretiondisks,"Astrophys. Journal, vol. 439, pp. L39-L42, 1995

[15] A. C. S. Readhead,G. B. Taylor,W. Xu, T. J. Pearson,P. N. Wilkinson, A. G. Polatidis,"The statistics andages of compact symmetric objects,”The Astrophy.Journ., vol. 460, pp. 612-633, 1996

[16] J. C. Cavalho, "The evolution of GHz-peaked-spectrumradio sources," Astronomy and Astrophysics, vol. 329,pp. 845-852, 1998

[17] F. Santoro, J. B. R. Oonk, R. Morganti, and T. Oosterloo, "The jet-ISM interaction in the outer filament of Centaurus A,"Astron. and Astrophy., vol. 574, A89, 2015

[18] J. C. Ezeugo,andA. A. Ubachukwu, "The spectralturnover-linear size relation and the dynamical evolution of compact steep spectrum sources", Mon. Not.Roy Ast. Soc, vol. 408, pp.2256-2260,2010

[19] I. A. G. Snellen,R. T.Schilizzi,G. K. Miley,M. N.Bremer,H. J. A. Rottgering, andH. J. van Langevelde,"Faint gigahertz peaked spectrum sources and the evolution of young radio sources,” New Ast. Rev., vol. 43,pp. 675-679, 1999

[20] I. Owsianik, andJ. E. Conway, "First detection ofhotspot advance in a compact symmetric object" Astron. and Astrophy., vol. 337, pp. 69-79, 1998

[21] W. Tschager,R. T.Schilizzi,I. A. G.Snellen,A. G. deBruyn, G. K. Miley, H. J. A. Rottgering, H. J. van Langevelde,C. Fanti,and R.Fanti, "VSOP/global VLBIobservations of 2021+614: detection of hotspot advance," New Ast. Rev., vol. 43, pp. 681-684, 1999

[22] C. P. O'Dea, R. A. Dally, P. Kharb, andK. A. Freeman,"Physical properties of very powerful FRII radio galaxies", Astro. and Astrophys., vol. 494, pp. 471, 2009

[23] F. Mantovani,W. Junior,M.Bondi,W. Cotton,R. Fanti,L.Padrielli,G. D. Nicolson,and E. Salerno,"Largebent jets in the inner region of CSSs," Astron. andAstrophy., vol. 332, pp. 10-18, 1998

[24] S. Jeyakumar,P. J.Wiita, D. J.Saikia, andJ. S.Hooda, “Jet propagation and the asymmetries of CSS radiosources," Astron. and Astrophy., vol. 432, pp. 823-833,2005

[25] A. Labiano, "Tracing jet-ISM interaction in youngAGN: correlations between [O III] $\lambda 500 \dot{A}$ and 5GHzemission", Astron. and Astrophy., 488, vol. L59-L62,2008 